\title{
Real-Time Speed Control of an Electrically Powered Wheelchair by Using Fractional Order Model Reference Adaptive System Control
}

\author{
Kagan Koray Ayten \\ Department of Electrical \& Electronics Engineering, Erzurum Technical University, \\ Yakutiye, 25700, Erzurum, Turkey \\ kagan.koray@erzurum.edu.tr
}

\begin{abstract}
In this study, speed and direction angle control technique based on the fractional order model reference adaptive system (FO-MRAS) are proposed for controlling an electrically powered wheelchair (EPW) system for the first time in the literature. The model reference adaptive system (MRAS) technique has been used to achieve the estimated value of any system's velocity information. In general, the control of the MRAS technique is usually performed by the PI based controller. However, the classical PI controllers are unable to meet the demands of high precise trajectory control during the motion. In this study, to increase the effectiveness of the PI based MRAS controller, this technique is combined with a fractional-order (FO) calculus to obtain an accurate and a robust trajectory tracking performance for an EPW. The proposed FO-MRAS controller has been compared experimentally with the conventional PI based MRAS method to show the effectiveness of using FO-MRAS method in terms of trajectory tracking accuracy and error levels. The experimental outcomes demonstrate that the proposed FOMRAS controller gives a better trajectory tracking performance as well as having a smaller speed error.
\end{abstract}

Index Terms-Fractional order PID; Nonlinear adaptive control; PMSM MRAS control; Trajectory control.

\section{INTRODUCTION}

In recent years, a robust control system for an automated guided vehicle (AGV) has been attracting a great attention because of its dynamic nonlinearities and uncertainties in the system. In contrast to robotic manipulators, the AGV is the non-holonomic systems due to the rolling constraints in the wheels. These constraints make kinematic and dynamic analyses more difficult than those of holonomic systems. Therefore, significant effort in a variety of solutions to the problem of mobile robot control has been investigated by the robotics research community. In addition to the AGVs, one of the popular research topics of recent times is smart wheelchairs which are also non-holonomic structure such as AGVs. Most of the smart wheelchair platforms are actuated by using electrical motors and theirs drivers. As wellknown, if the position and velocity of the electric motor can be controlled accurately than the trajectory tracking control of the system will be precise. When the wheeled mobile robot or an EPW are considered, the dynamic model of the system has highly time-varying nonlinearities due to un-

Manuscript received 3 January, 2018; accepted 7 April, 2018. modelled system dynamics and undesirable disturbance effects. The most controllers designed for the nonlinear control of wheeled mobile robots are based on the kinematics of the system [1]-[8]. However, it is not realistic to control the mobile robot or smart EPW in the real world by considering only the kinematic model due to the uncertainties in their modelling. Hence, it becomes indispensable to take into account the EPW's dynamics in addition to its kinematics, when heavy load carrying and high speed movement are required. In such cases, it is pretty essential to design a controller with great efficiency to run the system effectively.

In literature, implementation of the velocity control of a mobile robot or/and an EPW can be divided into two main control approaches. The first approach utilizes information about the actuator speed for closed-loop control. Therefore, position sensors need to be mounted onto the actuator, and many research efforts have been made for the speed control by using position sensors [9]-[11]. The second approach is focused on how to observe the velocities of the actuator with elimination of the position sensors. Without affecting the system performance, to eliminate the actuator speed sensor on the actuator, is an important approach in the advanced control system for drives. Using an actuator mounted speed sensors increases the cost of the system and decreases temperature sensitivity and reliability. On the other hand, if the system runs without speed sensor, hardware complexity can be reduced and better noise immunity and higher robustness can be provided during the motion. In the direction indicated above, in literature, to calculate the actuator position and the speed of the mobile robot, many researchers have performed the Extended Kalman Filter (EKF) in their study [12], [13]. However, the Kalman Filter approach has some inherent disadvantages, such as a large computational complexity and the influence of noise. And also many methods are used and generally accepted due to provide high sensorless performance, such as sliding mode observers, an adaptive non-linear flux observer, and different improvements [14], 15]. In general, the control of the sensorless model reference adaptive system (MRAS) is usually performed by the classical PI controller. However, the classical PI controllers are unable to meet the demands of high precise trajectory control during the motion. Therefore, in this study, to overcome the aforementioned 
problems above, the fractional order control action is combined with the sensorless PI based MRAS technique for an EPW, which is actuated by a permanent magnet synchronous motor (PMSM). The performance of the fractional control technique is robust to uncertainties in robotic systems due to the technique has a parameter-based structure. The fractional order PID (FO-PID) controllers have become a popular technique recently in control applications [16], [17]. Fractional-order (FO) is an arbitrary order of the ordinary derivative and integral calculus that leads to more exibility and performance in the designing of the controller.

In this study, the PMSM motor which is actuating system is analysed firstly due to the relationship between the performance of driving and effectiveness of trajectory tracking. Secondly, the implementation and performance evaluation of sensorless vector control of three-phase PMSM motor is carried out in this paper. The MRAS technique is used for the estimation of motor speed. Finally, motivated by the advantages of the fractional order control technique, a method that combine the fractional order control action with the MRAS technique to improve the trajectory tracking precision and the robustness of the system. To demonstrate the effectiveness of the proposed FO-MRAS method, the real time studies have been executed and compared with the conventional PI-based MRAS method.

The rest of the paper structure is given as follows: the mathematical modelling of an EPW and 3-Phase PMSM are given in section II. The explanation of the control strategies such as vector control of 3-phase PMSM, FO, MRAS based speed estimation control are described in Section III, the experimental results are given in Section IV, finally, the discussion and conclusion are presented in Section $\mathrm{V}$ and Section VI.

\section{MATHEMATICAL Modelling}

In this section, the mathematical modelling of an EPW platform and also 3-Phase permanent magnet synchronous motor have been presented.

\section{A. Mathematical Modelling of an EPW Platform}

As shown in Fig. 1, the motion and the orientation of the mobile robot are run by actuators of the wheels, and each actuator is independent and actuated by a PMSM.

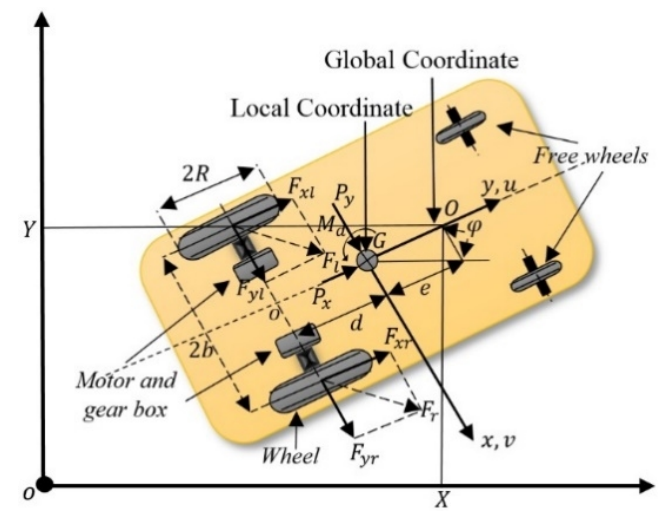

Fig. 1. 2 D Model of an EPW (mobile robot)

For the purpose of analysis, two coordinate systems are defined. The $O-X Y$ defines the global coordinate system which is fixed to the Cartesian workspace and the $G-x y$ describes the local coordinate system that attached to the mobile robot's centre of mass. The longitudinal and lateral velocities at the centre gravity of the mobile robot platform are shown by $u$ and $v$. Also, $r$ is the yaw rate, the right and left wheel angular velocities are given by $\omega_{r}$ and $\omega_{l}$, respectively. About the conditions of slipping, we can write the following kinematic equations in velocity dimension:

$$
\begin{gathered}
u=\frac{1}{2}\left(u_{l}+u_{r}\right), \\
v=d r+v^{s}, \\
r=\frac{1}{2 b}\left(u_{l}-u_{r}\right), \\
u_{l}=R \omega_{l}-u_{l}^{s}, \\
u_{r}=R \omega_{r}-u_{r}^{s} .
\end{gathered}
$$

The longitudinal velocity of the centres of the right and left wheels is given by $u_{r}$ and $u_{l}$, respectively. The wheel slip model can be taken into consideration by adding the three speed components $\left(v^{s}, u_{r}^{s}, u_{l}^{s}\right)$ into the kinematic model. In the absence of wheel slippage, the kinematic model can easily be obtained by adjusting above three components to zero. The distance from the mass centre of the platform to the wheel centre is given by $d$ and the distance from the geometric centre mark of the mobile robot structure to the wheel centre is given by $b . R$ is the radius of driving wheels. In the global coordinate $O-X Y$, the heading angle of the system is given as follows:

$$
\begin{gathered}
\dot{X}=u \cos \varphi-(v+e r) \sin \varphi, \\
\dot{Y}=u \sin \varphi+(v+e r) \cos \varphi, \\
\dot{\varphi}=r .
\end{gathered}
$$

The Newton's Law is used to create the equation of motion of the system and the Newton's Law can be found by summing the external moments and forces in the body cantered reference frame as follows:

$$
\begin{gathered}
F_{x r}+F_{x l}+P_{x}=m(\dot{u}-v r), \\
F_{y r}+F_{y l}+P_{y}=m(\dot{v}+u r), \\
\left(F_{x l}-F_{x r}\right) b-\left(F_{y l}+F_{y r}\right) d-M_{d}=I_{z} \dot{r}, \\
n \tau_{R}-F_{x r} R=I_{e} \omega_{r}, \\
n \tau_{L}-F_{x l} R=I_{e} \omega_{l},
\end{gathered}
$$

where $P_{x}, P_{y}$ and $M_{d}$ present the external forces and moment values at the mobile robot mass centre, respectively. The wheel forces (such as friction forces) on the driving right and left wheels are given by $\left(F_{x r}, F_{y r}\right)$ and $\left(F_{x l}, F_{y l}\right)$, respectively. The mass is given by $m$, and 
inertia of the robot is given by $I_{z}$ about $z$ axis, $n$ is the gear ratio. The wheel and the driving motor inertias $I_{t}$ and $I_{m}$ are combined to create the effective moment of inertia as expressed by $I_{e}=I_{t}+n^{2} I_{m}$. The right and left wheel's torque inputs are described by $\tau_{R}$ and $\tau_{L}$. As it is seen from (13), the desired trajectory tracking control of the system can be realised by means of controlling or changing supply voltage of the PMSM motors. The tire slip and angle are easily calculated by using $u, v, r, \omega_{r}$ and $\omega_{l}$, and effects of the tire slip and angle are lateral and also longitudinal forces as demonstrated in Fig. 1.

\section{B. Mathematical Modelling of 3-Phase PMSM}

For the considered and studied mobile robot, the EPW's right and left wheels are driven by two PMSM with two gear speed reducer. In speed control applications, the 3- phase PMSM is powered by means of voltage source inverters (VSI). The VSI is controlled by Pulse Width Modulation (PWM) technique as shown in Fig. 2.

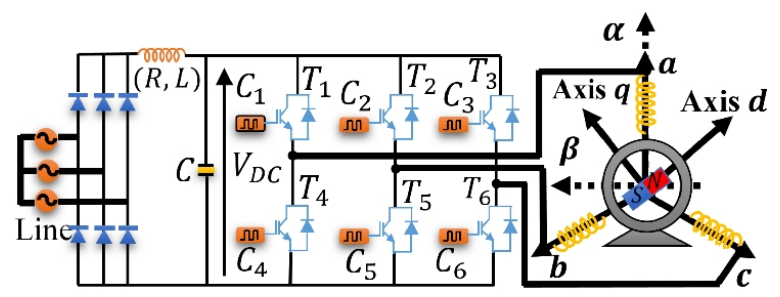

Fig. 2. Voltage source inverter fed 3- Phase PMSM.

In this figure, the output voltages $u_{s a}, u_{s b}$ and $u_{s c}$ fed to the star-connected PMSM windings and the DC voltage input inverter is shown by $V_{D C}$. The six Insulated-Gate Bipolar Transistor (IGBT) component's power module from $T_{1}$ to $T_{6}$ shape the output signals by means of switching signals $C_{1}$ to $C_{6}$. In order to describe the PMSM mathematically, the voltage equations of PMSM of the EPW are given in the time domain as follows:

$$
\begin{aligned}
& u_{s a}=R_{s} i_{s a}+\dot{\Psi}_{s a}, \\
& u_{s b}=R_{s} i_{s b}+\dot{\Psi}_{s b}, \\
& u_{s c}=R_{s} i_{s c}+\dot{\Psi}_{s c} .
\end{aligned}
$$

In these equations, the instantaneous values of stator voltages and currents are shown by $u_{s a}, u_{s b}$ and $u_{s c}$, and $i_{s a}, i_{s b}$ and $i_{s c}$, respectively, and $\Psi_{s a}, \Psi_{s b}$ and $\Psi_{s c}$ define the instantaneous values of stator flux linkages in phases $s a, s b$ and $s c$, respectively. The instantaneous form in (16) consists of the large number of equations. Therefore, PMSM can be expressed as more practical way of rewriting the instantaneous equations by means of using the two-axis theory (Clarke transformation) as follows:

$$
\begin{gathered}
u_{s \alpha}=R_{s} i_{s \alpha}+\dot{\Psi}_{s \alpha}, \\
u_{s \beta}=R_{s} i_{s \beta}+\dot{\Psi}_{s \beta}, \\
\Psi_{s \alpha}=L_{s} i_{s \alpha}+\Psi_{M} \cos \left(\theta_{r}\right), \\
\Psi_{s \beta}=L_{s} i_{s \beta}+\Psi_{M} \sin \left(\theta_{r}\right),
\end{gathered}
$$

$$
\dot{\omega}_{r}=\frac{p}{J}\left\{\frac{3}{2} p\left(\Psi_{s \alpha} i_{s \beta}-\Psi_{s \beta} i_{s \alpha}\right)-T_{L}\right\},
$$

where $\alpha, \beta$ are the stator orthogonal coordinate system, $u_{s \alpha}, i_{s \alpha}$ and $u_{s \beta}, i_{s \beta}$ are the stator voltages and currents in $\alpha, \beta$ axes, respectively. The stator magnetic flux and the rotor magnetic flux can be demonstrated by $\Psi_{s \alpha}, \Psi_{s \beta}$ and $\Psi_{M}$, respectively. The stator phase inductance and the stator phase resistance are shown by $L_{S}$ and $R_{S}$, respectively. The $\omega_{r}$ describes the electrical rotor speed, $p$ and $J$ are the number of poles per phase and the inertia, respectively. The load torque is given by $T_{L}$ and $\theta_{r}$ is the rotor position in $\alpha, \beta$ axes. In this study, the vector control algorithm is performed for the speed control of the EPW. To make the control of PMSM easier and effectively, the vector control is performed entirely in the $d, q$ coordinate system instead of using $\alpha, \beta$ coordinate system. Using the park transformation ( $\alpha, \beta$ to $d, q)$, the PMSM model can be obtained as follows:

$$
\begin{gathered}
u_{s d}=R_{s} i_{s d}+\dot{\Psi}_{s d}-\omega \Psi_{s q}, \\
u_{s q}=R_{s} i_{s q}+\dot{\Psi}_{s q}-\omega \Psi_{s d}, \\
\Psi_{s d}=L_{s} i_{s d}+\Psi_{M}, \\
\Psi_{s q}=L_{s} i_{s q}, \\
\omega_{r}=\frac{p}{J}\left\{\frac{3}{2} p\left(\Psi_{s q} i_{s q}-\Psi_{s q} i_{s d}\right)-T_{L}\right\} .
\end{gathered}
$$

In the rotor reference frame, the axes stator currents ( $q$ and $d$ ) can be obtained as

$$
\left[\begin{array}{c}
i_{s q} \\
i_{s d}
\end{array}\right]=i_{s}\left[\begin{array}{c}
\sin \delta \\
\cos \delta
\end{array}\right]
$$

Moreover, (24) and (25) can be substituted into (22) and (23), the vector and matrix forms of dynamic equation of the PMSM are given as follows

$$
U=[R] i+[L] \partial i+[G] \omega i
$$

In (28), the resistive elements are included in $[R]$ matrix, the derivative operator's coefficients, $\partial$, is included in $[L]$ matrix, and also the electrical rotor speed's coefficient, $\omega_{r}$ is included in the $[G]$ matrix. The mechanical dynamics of the machine can be determined by the most important output variable which is the electromagnetic torque. This electromagnetic torque is calculated using machine matrix equations. When this calculation is made, various components such as the input power of the system and the amount of change of the stored magnetic energy are taken into account. Similarly, in a steady state situation, the amount of stored magnetic energy is zero. For this reason, the output power can be shown as the difference between 
the resistive losses and the input power. In order to compute the instantaneous input power as in (29), the both sides of (28) is multiplied by the current vector's transpose as follows

$$
P_{\text {input }}=i^{T}[U]=i^{T}[R] i+i^{T}[L] \partial i+i^{T}[G] \omega_{r} i
$$

In (29), the rotor and the stator resistive losses are shown by the term of $i^{T}[R] i$, and the term $i^{T}[L] \partial i$ denotes the amount of change magnetic energy. Hence, the remainder of the power component should be equivalent to the term $i^{T}[G] \omega_{r} i$ which is the air gap power. It is clearly known that, there is a close connection between the rotor speed and the air gap power, therefore, the air gap torque, $T_{e}$, can be derived from the rotor speed $\omega_{m}$, which is in mechanical $\mathrm{rad} / \mathrm{s}$, as follows

$$
\omega_{m} T_{e}=i^{T}[G] i x \omega_{r}=i^{T}[G] i \frac{p}{2} \omega_{m} .
$$

In (30), the number of poles is shown by $p$ and if the speed is cancelled on both sides, $T_{e}$ becomes as shown as follows

$$
T_{e}=\frac{p}{2} i^{T}[G] i
$$

Substituting $[G]$ in (31) with the observation from (28), the electromagnetic torque can be obtained as follows

$$
T_{e}=\frac{3}{2} \frac{p}{2}\left[\Psi_{M}+\left(L_{d}-L_{q}\right) i_{s d}\right] i_{s q},[N . m] .
$$

Among the two-phase and three-phase PMSM, from the power equivalence condition, the value of $3 / 2$ can be presented on the right-hand side of (32). Then, the normalized electromagnetic torque, $T_{e}$, in per unit can be found as follows [18], [19]

$$
T_{e_{n}}=i_{s q_{n}}\left[\Psi_{M_{n}}+\left(L_{d_{n}}-L_{q_{n}}\right) i_{s d_{n}}\right],[p . u] .
$$

In (33), the electromechanical dynamic equation is also described as follows [18]

$$
T_{e}=J \omega_{m}+T_{L}+B \omega_{m}
$$

In this equation, the mechanical rotor speed and the friction coefficient are shown by $\omega_{m}$ and $B$, respectively. Load torque value, $T_{L}$, in (34) should be equal to one of $n \tau_{R}$ or $n \tau_{L}$, given in (13) for the considered PMSM in order to obtain actuator torques. On the other hand, in Fig. 3, the vehicle drive system for the $d-q$ axis is shown in the schematic of the PM synchronous motor.

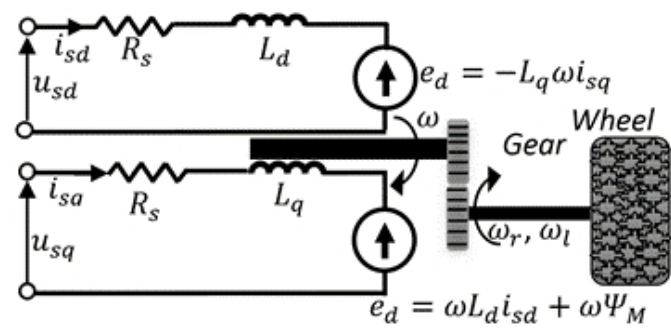

Fig. 3. The vehicle drive system for the $d q$-axis of PMSM.

By considering the gear ratios $(n)$ as shown in Fig. 3, angular rotor speed of the output shaft of PM synchronous motor $\left(\omega_{m}\right)$ will be equivalent to the angular speed of the right or left wheels $\left(\omega_{r}, \omega_{l}\right)$ by introducing the following expression

$$
n=\frac{\omega}{\omega_{r}}=\frac{\omega}{\omega_{l}}
$$

\section{CONTROL STRATEGIES}

\section{A. Vector Control of 3-Phase PMSM}

Utilising the vector of PMSM lets similar dealings with the motor as with a DC separately induced DC machine. The $d$ and $q$ inductances of the surface mounted PMSM are equal $L_{d}=L_{q}$. In this case, normalized electromagnetic torque can be shown per unit as follows [18]-[20]

$$
T_{e_{n}}=\Psi_{M_{n}} i_{s q_{n}},[p . u] \text {. }
$$

By using (27), the torque can be written as follows [18]

$$
T_{e}=\Psi_{M} i_{s} \sin \delta
$$

The torque gets maximum rate for $\delta$ equivalent to 90 degrees for a certain value of the stator current. This delivers maximum torque for per ampere and hence efficiency will be high [18]-[20]. Keeping $i_{s d}$ zero is the most well-known control method for PMSM. This method helps the machine to be protected against under-or-excited conditions. The basic structure of PMSM vector control is demonstrated in Fig. 4.

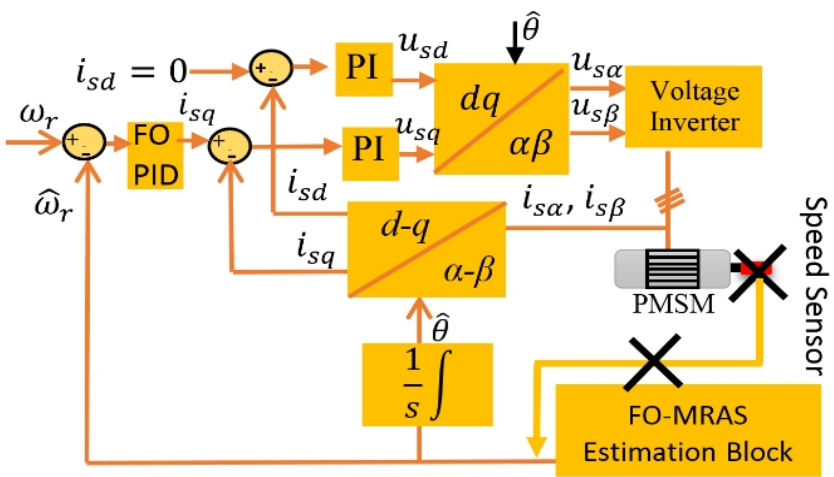

Fig. 4. Basic structure of PMSM vector control.

To perform vector control, these steps need to be followed; the motor quantities such as phase voltages, 
currents and angular rotor speed of motor need to be measured, these quantities has to be transformed into a 2phase system $(\alpha, \beta)$ by utilising Clarke transformation and then transform stator currents into the $d-q$ coordinate system by means of Park transformation. The stator current torque $i_{s q}$ and flux $i_{s d}$ creating components are individually controlled by means of the controllers. The stator voltage space vector can be converted back from the $d-q$ coordinate into the two-phase system.

\section{B. MRAS Based Speed Estimation}

To predict the rotor speed and the stator $d-q$ axis currents, the FO-MRAS estimators are designed. In this design, the speed sensor is bypassed. The main idea of this method is that the MRAS method is an adjustable model and a reference. Where the first one is utilized so as to detect the necessary states and the second one as an adaptive model ensure the estimated values of the states.

In this system, an adjustable observer-based model is compared the outcome of the reference model in order to obtain the error between the adjustable and the reference models. This error is then forwarded to a FO adaptation mechanism which adjusts the adaptive model by producing an estimated value of the rotor speed. The block diagram of the estimation technique based on the MRAS method is given in Fig. 5. Using (22), the following expression can be obtained as follows [18]:

$$
\begin{gathered}
\psi_{s d}=u_{d}-R_{s} i_{d}+\omega_{r} \psi_{s q}= \\
=u_{d}-R_{s}\left(\frac{\psi_{s d}-\psi_{f}}{L_{d}}\right)+\omega_{r} \psi_{s q}= \\
=-\frac{R_{s}}{L_{d}} \psi_{s d}+\omega_{r} \psi_{s q}+\frac{R_{s}}{L_{d}} \psi_{f}+u_{d}, \\
\psi_{s q}=u_{q}-R_{s} i_{q}-\omega_{r} \psi_{s d}= \\
=u_{d}-R_{s}\left(\frac{\psi_{s q}}{L_{q}}\right)-\omega_{r} \psi_{s d}= \\
=-\frac{R_{s}}{L_{q}} \psi_{s q}-\omega_{r} \psi_{s d}+u_{q} .
\end{gathered}
$$

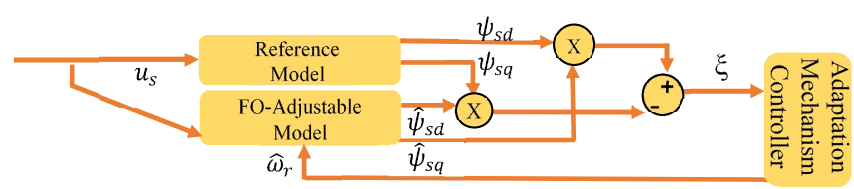

Fig. 5. Block diagram of the estimation technique with FO-MRAS.

Therefore, the observer equation is appeared as:

$$
\begin{gathered}
\hat{\psi}_{s d}=-\frac{\hat{R}_{s}}{L_{d}} \hat{\psi}_{s d}+\hat{\omega}_{r} \hat{\psi}_{s q}+\frac{\hat{R}_{s}}{L_{d}} \psi_{f}+u_{d}+k_{d}, \\
\hat{\psi}_{s q}=-\frac{\hat{R}_{s}}{L_{d}} \hat{\psi}_{s q}-\hat{\omega}_{r} \hat{\psi}_{s d}+u_{q}+k_{q}, \\
i_{d}=\frac{\hat{\psi}_{s d}}{L_{d}}+\frac{\psi_{f}}{L_{d}},
\end{gathered}
$$

$$
i_{q}=\frac{\hat{\psi}_{s q}}{L_{q}}
$$

In (43), the control signals, $k_{d}$ and $k_{q}$, are designed based on the back-stepping theory. So, the obtained error between the observed and the real values are given as follows:

$$
\begin{gathered}
\tilde{\psi}_{s d}=\psi_{s d}-\hat{\psi}_{s d}, \\
\tilde{\psi}_{s q}=\psi_{s q}-\hat{\psi}_{s q}, \\
\tilde{\omega}_{r}=\omega_{r}-\hat{\omega}_{r}, \\
\tilde{R}_{s}=R_{s}-\hat{R}_{s} .
\end{gathered}
$$

Then the error response can be represented by:

$$
\begin{gathered}
\tilde{\psi}_{s d}=-\frac{\tilde{R}_{s}}{L_{d}} \tilde{\psi}_{s d}+\tilde{\omega}_{r} \tilde{\psi}_{s q}+\frac{\tilde{R}_{s}}{L_{d}} \psi_{f}+u_{d}+k_{d}, \\
\tilde{\psi}_{s q}=-\frac{\tilde{R}_{s}}{L_{d}} \tilde{\psi}_{s q}-\tilde{\omega}_{r} \tilde{\psi}_{s d}+u_{q}+k_{q}, \\
i_{d}=\frac{\tilde{\psi}_{s d}}{L_{d}}+\frac{\psi_{f}}{L_{d}}, \\
i_{q}=\frac{\tilde{\psi}_{s q}}{L_{q}} .
\end{gathered}
$$

The speed tuning signal actuates the rotor speed, that is, the rotor speed converges the error signal to zero. In this study, the fractional order PID controller algorithm is used by the adaptation mechanism of the MRAS-based speed estimation method as follows:

$$
\begin{gathered}
\hat{\omega}_{r}=K_{p} \xi+K_{i} \int \xi+K_{d} \frac{d \xi}{d t}, \\
\xi=\psi_{s d} \hat{\psi}_{s q}-\psi_{s q} \hat{\psi}_{s d},
\end{gathered}
$$

where the $\hat{\omega}_{r}$ is a controller, and $K_{p}, K_{i}$ and $K_{d}$ are the controller gains and $\xi$ is the error signal. It is an obvious fact that, $\hat{\omega}_{r}$ that appears in (53), is now the predict speed of our right and left wheels. In this case, we can easily estimate longitudinal and lateral velocities at the centre gravity of the mobile robot platform.

\section{Fractional Oder Controller Design}

In this study, the fractional-order $P I^{\lambda} D^{\mu}$ controller algorithm has been performed for the adaptation mechanism of the MRAS-based speed estimation method to increase the trajectory tracking accuracy during the estimation. The most frequently used the fractional derivative definitions is the Riemann-Liouville definition. In time domain, the Riemann-Liouville $(R L)$ fractional derivative and integral of $\lambda_{t h}$ order of function $f(t)$ with respect to $t$ and the terminal value $a$ is given by:

$D_{a t}^{\lambda} f(t)=\frac{d^{\lambda} f(t)}{d t^{\lambda}}=\frac{1}{\Gamma(r-\lambda)} \frac{d^{r}}{d t^{r}} \int_{a}^{t} \frac{f(\tau)}{(t-\tau)^{\lambda-\mathrm{r}+1}} d \tau$, 
$D_{a t}^{-\lambda} f(t)=L_{a t}^{\lambda} f(t)=\frac{1}{\Gamma(\lambda)} \frac{d^{r}}{d t^{r}} \int_{a}^{t} \frac{f(\tau)}{(t-\tau)^{\lambda-\mathrm{r}+1}} d \tau$,

where $-1<\lambda<r$, while, $D^{\lambda}$ and $L^{\lambda}$ denote the fractional derivative and integral, respectively. And $\Gamma($.$) is Euler's$ Gamma function given by

$$
\Gamma(\lambda)=\int_{0}^{\infty} e^{-t} t^{\lambda-1} d t
$$

Using the Riemann-Liouville ( $R L$ ) fractional derivative and integral of $\lambda_{t h}$ order of function $f(t)$, the fractionalorder $P I^{\lambda} D^{\mu}$ controller can be defined in Laplace form follows

$$
C_{c}(s)=K_{p}+K_{i} s^{-\lambda}+K_{d} s^{\mu}
$$

where $(\lambda, \mu>0)$.

The proportional, the integral and the derivative constants are given by $K_{p}, K_{i}$ and $K_{d}$, respectively. As it is seen from (57), the controller becomes the classical PID form for $\lambda=1$ and $\mu=1$. The performance of the $P I^{\lambda} D^{\mu}$ controller method for the considered system is much better than the conventional PID controller method due to its higher degrees of freedom for tuning. Hence, the adaptation mechanism of the MRAS-based speed estimation method uses the fractional-order $P I^{\lambda} D^{\mu}$ controller algorithm as follows

$$
\hat{\omega}_{r}=K_{p} \xi+K_{i} D^{-\lambda} \xi+K_{d} D^{\mu} \xi
$$

\section{EXPERIMENTS}

\section{A. Experimental Setup}

Figure 6 shows the designed electric-powered wheelchair system. The system has two rear driving wheels and two free caster front wheels. Each driving wheel has been coupled to the shaft of the PMSM motor and each PMSM motor is fitted with gearhead.

Moreover, the system has a personal computer with a controller board for real time applications. The proposed
FO-MRAS has been performed experimentally on an EPW. It has an external control box with two amplifiers and the controllers are commanding the PMSM and measuring the currents at a rate of $1 \mathrm{kHz}$ (Rapid prototyping technique was used [21]). Carrying load capacity is approximately $90 \mathrm{~kg}$. The experiments have been made by Matlab/Simulink software by using Runge-Kutta solver with a fixed-step of 0.002. Using the pattern search optimization algorithm, controller parameters are optimized. Depending on the desired reference values, the speed and direction angles that the vehicle must make, and the angular velocities of the right and left wheels accordingly, are calculated by a subprogram written in MATLAB. Then, the kinematics of the vehicle use these velocity values to calculate the angular reference velocities of the right and left wheels.

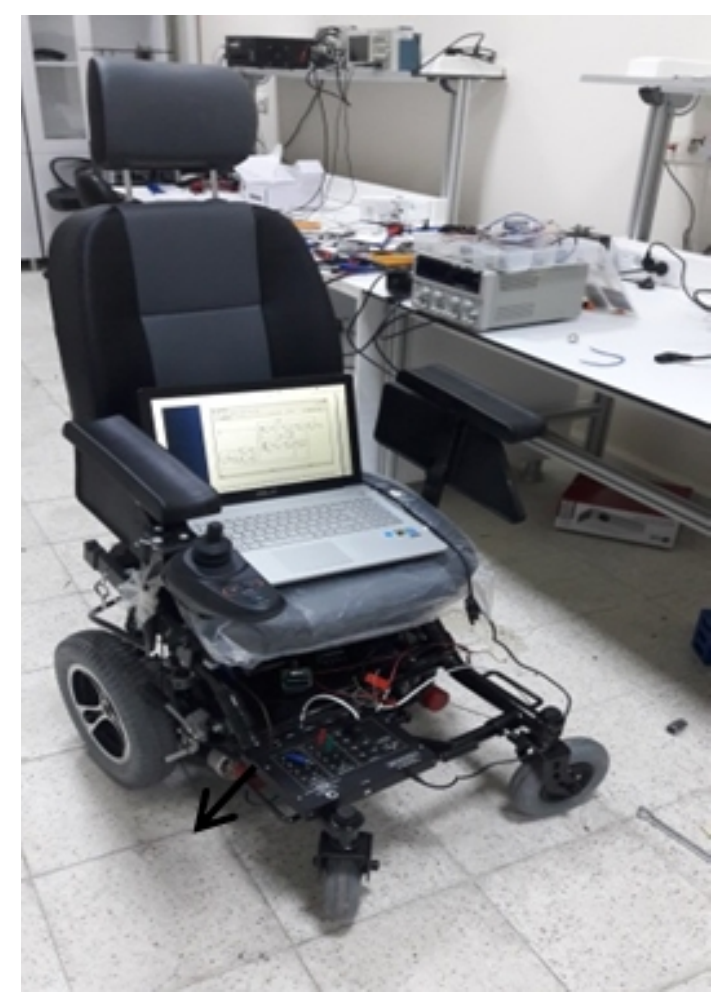

Fig. 6. Electric powered wheelchair.

These reference values are used as the FO-MRAS based vector control input as shown in Fig. 7. Depending on the controller parameters, the appropriate controller switching signals are sent to the voltage source inverter. At this point the desired angular velocity is reached.

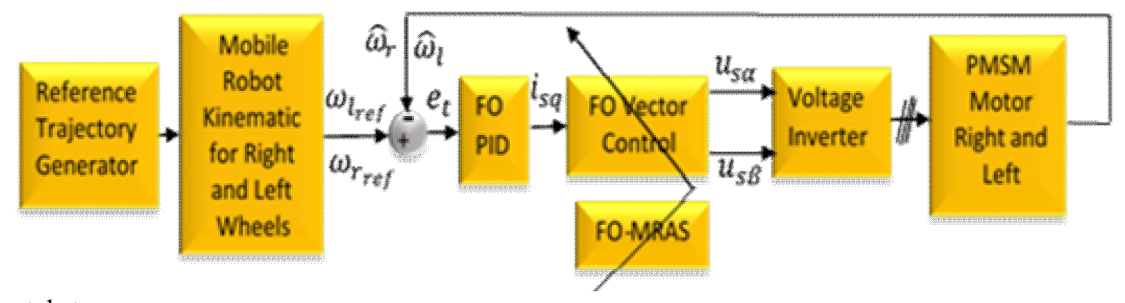

Fig. 7. Diagram of experimental step.

\section{B. Experimental Results}

After modelling the kinematic and dynamic model of EPW, PI-based MRAS and the proposed FO-MRAS controller have simulated experimentally with 3 different case studies in order to verify the effectiveness of the proposed FO-MRAS controller (Fig. 8-Fig. 19). Each case takes into account a different trajectory as follows:

Case Study 1

In this first case, a sinusoidal speed and direction angle references were used to test the effectiveness of the PI-based 
MRAS and the proposed FO-MRAS controller.

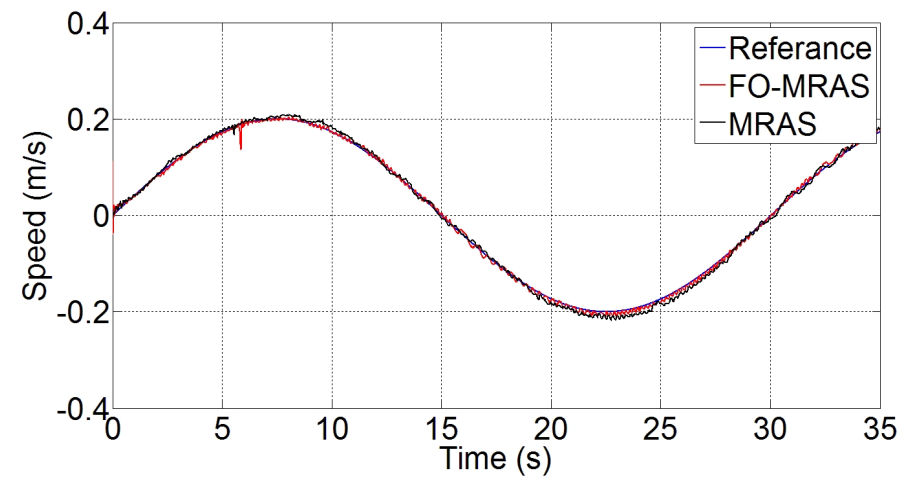

Fig. 8. Experimental result for the sinusoidal speeds.

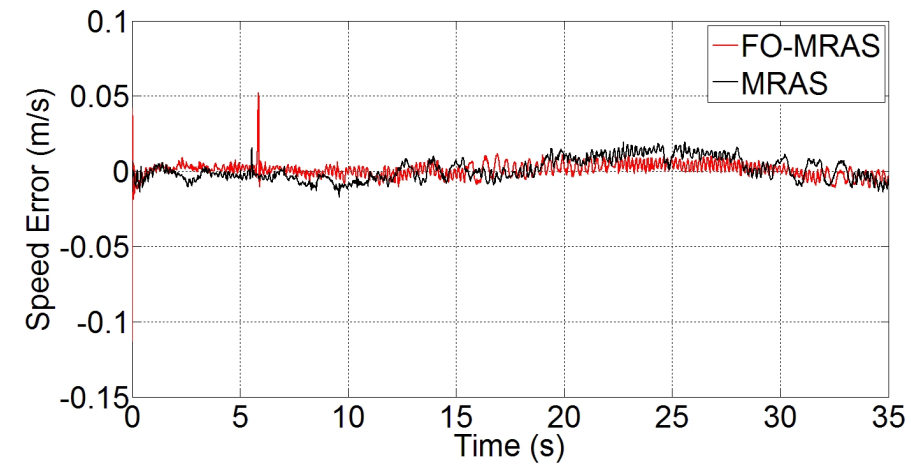

Fig. 9. Error for the sinusoidal speeds.

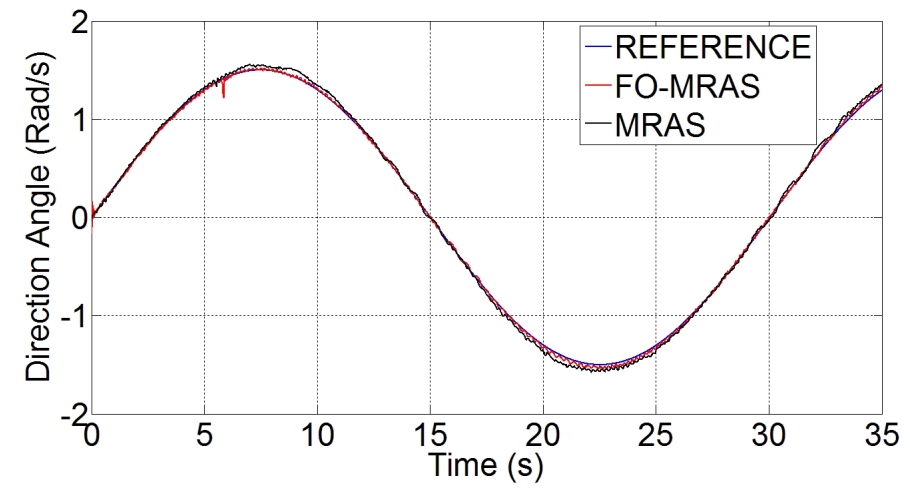

Fig. 10. Experimental result for the sinusoidal direction angle.

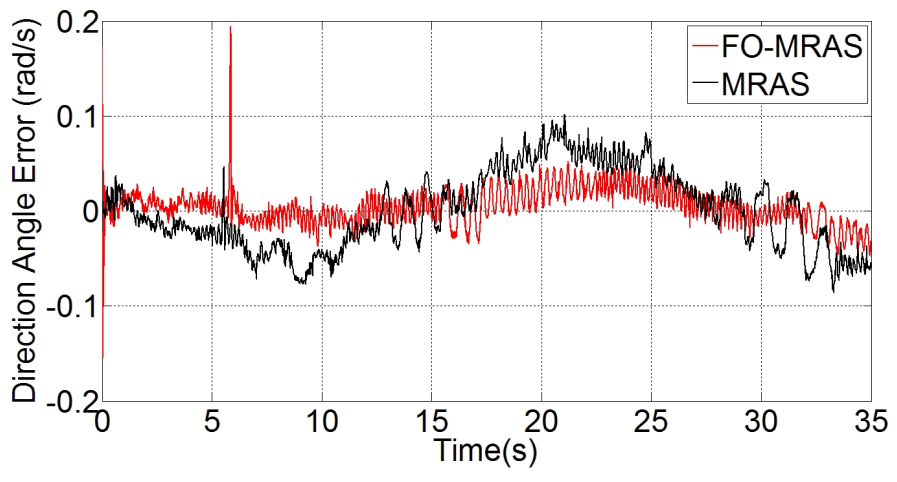

Fig. 11. Error for the direction angle.

Case Study 2

In response to another experiment case and in order to demonstrate the adaptation of the proposed FO-MRAS controller, a square wave reference speed trajectory was created. The sine function is chosen to create direction angle. In order to verify the effectiveness of the proposed controller for step changes, a square wave is crucial for almost every controller. 


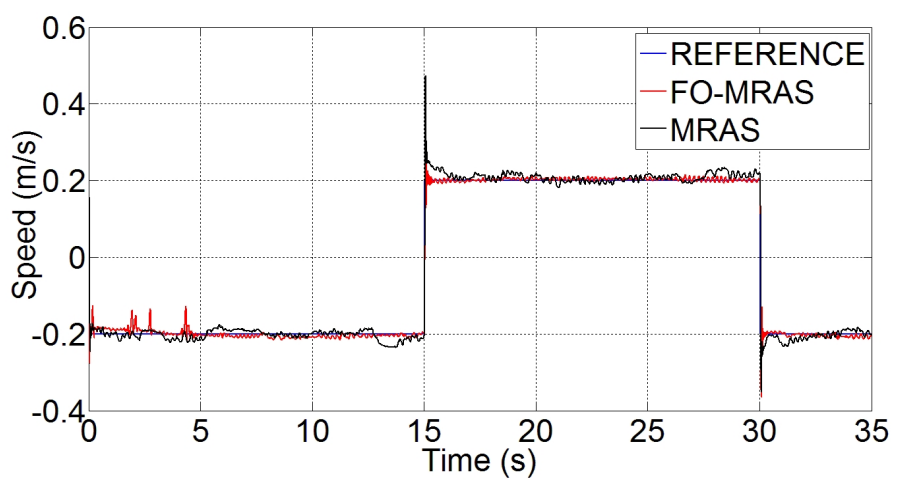

Fig. 12. Experimental result for the square speeds.

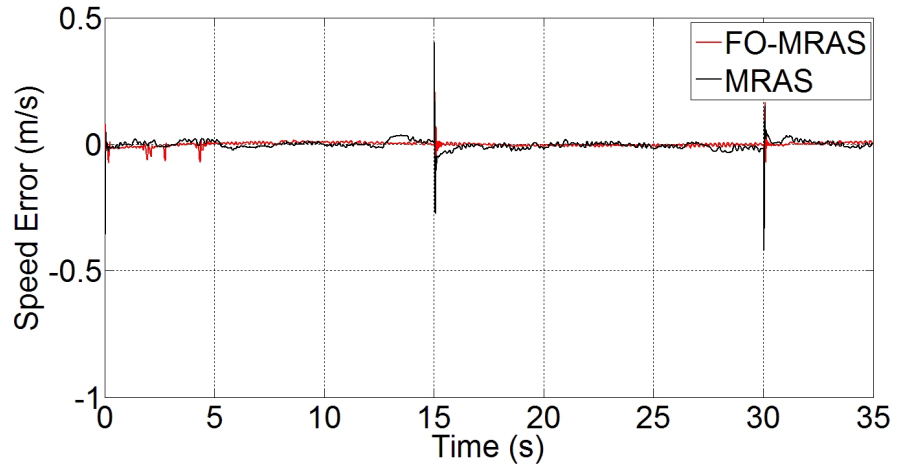

Fig. 13. Error for the square speeds.

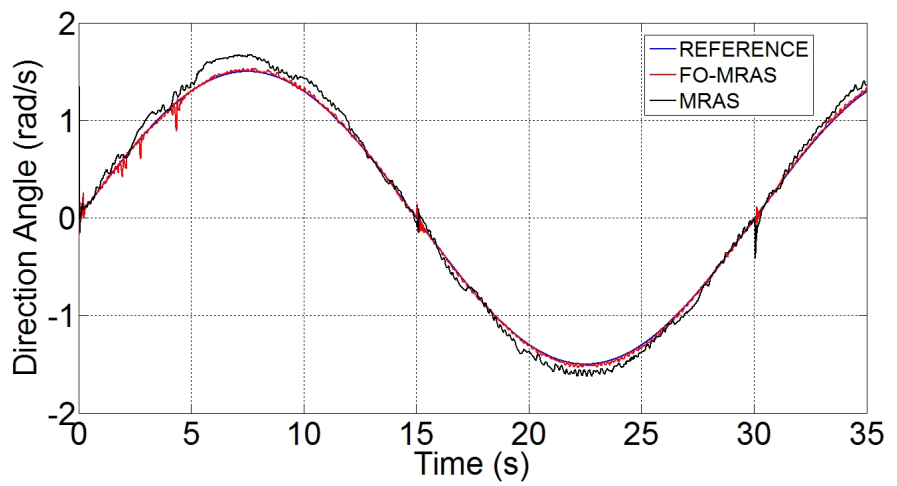

Fig. 14. Experimental result for the square direction angle.

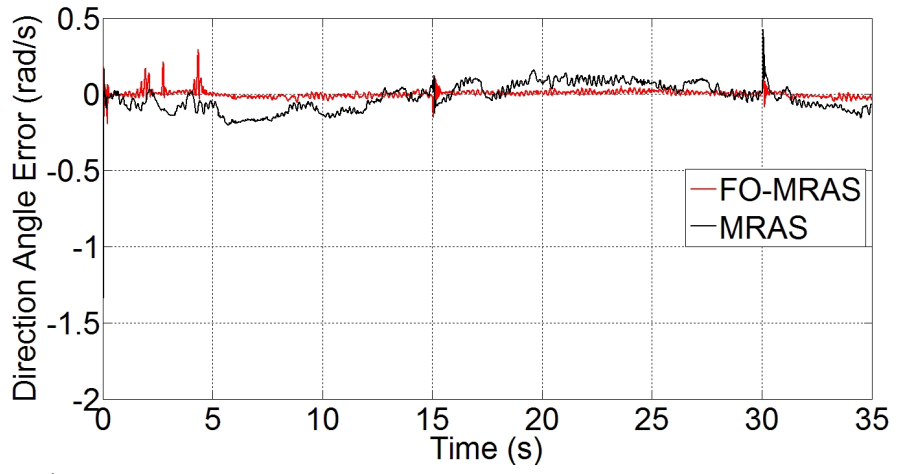

Fig. 15. Error for the square direction angle.

Case Study 3

In the final case, a triangle wave and sinusoidal direction angle references are used for the experimental study (Fig. 16-Fig. 19).

\section{DISCUSSION}

As can be seen from the figures of case studies, using the proposed FO-MRAS and PI-based MRAS control law, speed and directional angle tracking error have been effectively minimized. As seen in figures, the proposed FOMRAS provides quite a good tracking performance when compared to the PI-based MRAS controller due to its adaption and FO actions for given tracking tasks. Additionally, both transient and steady-state error values are decreased by utilizing the FO-MRAS controller. 


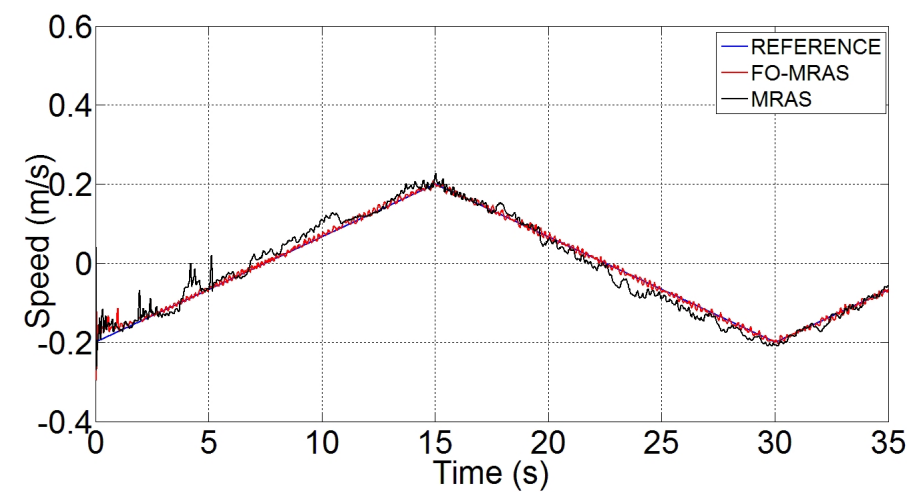

Fig. 16. Experimental result for the triangle speeds.

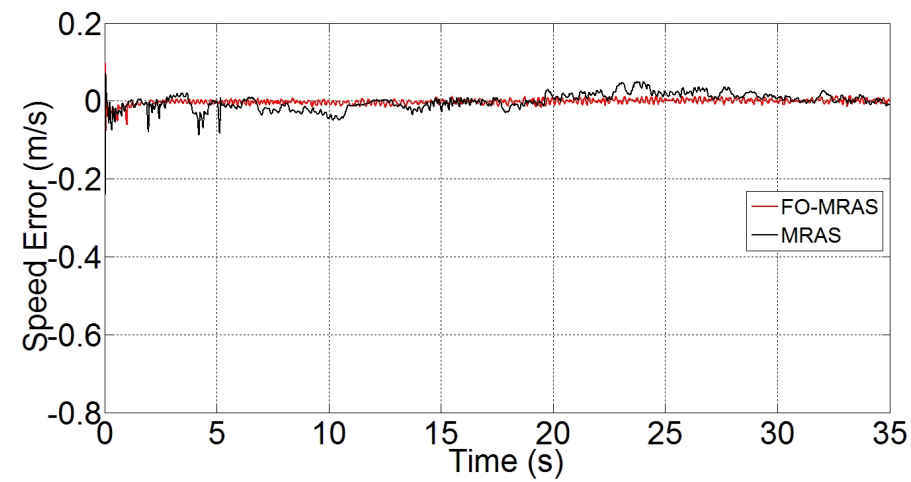

Fig. 17. Error for the triangle speeds.

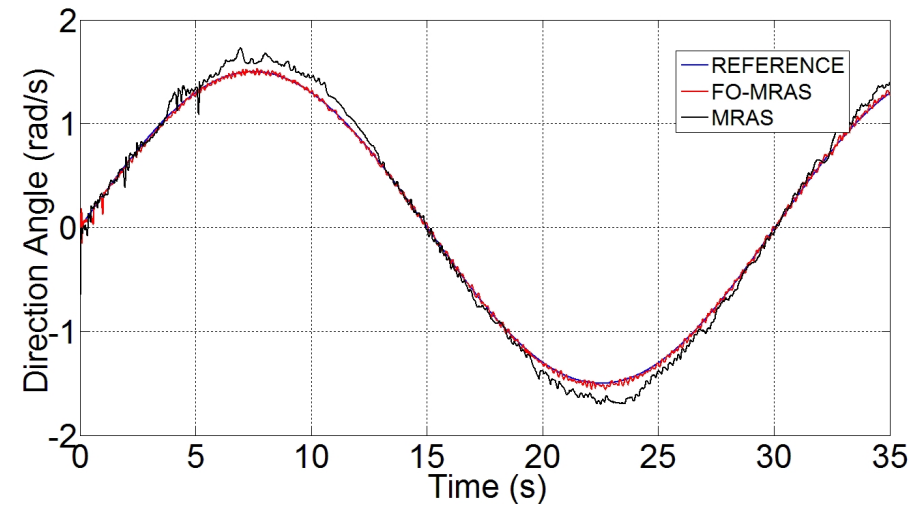

Fig. 18. Experimental result for the triangle direction angle.

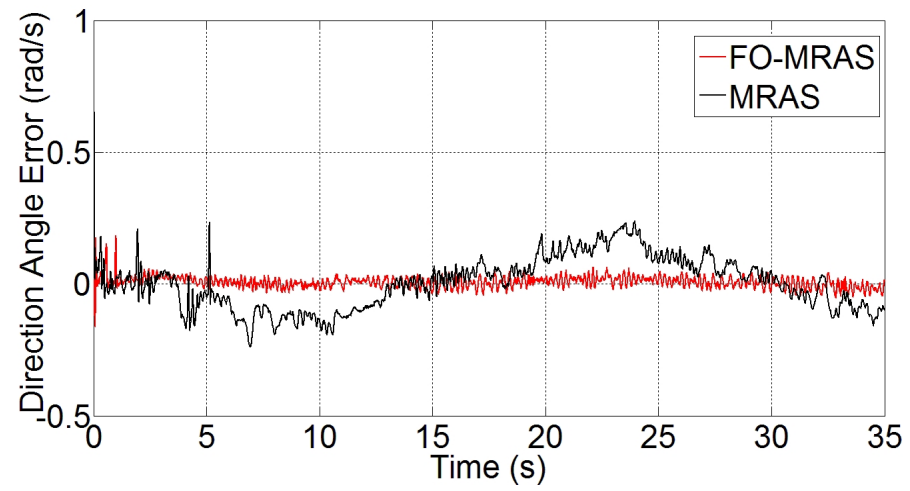

Fig. 19. Error for the triangle direction angle.

To show the efficiency of the proposed controller, the mean squared error (MSE) values of the proposed and conventional methods for given reference are calculated and tabulated in Table I. As tabulated in Table I, RMS values are seen to be less in the FO-MRAS when it is compared to the PI based MRAS control. In the first method, a PI based MRAS controller was used without taking into account fractional order control. In the second method, the fractional order technique is combined with the PI based MRAS technique and the control of the system is provided in this way. Experimental results have shown that the proposed FO-MRAS technique is adaptive to changing conditions and can respond very quickly to control the system's real speed and directional angle. When we evaluate the results, the 
control of velocity and direction angles is very successful both in the PI-based MRAS method and in the proposed FOMRAS technique, even though the EPW platform is sensorless and loaded. However, the FO-MRAS technique seems to perform the reference trajectory quite well than the MRAS technique. The reason for the fluctuations occurring during the EPW's trajectory tracking is due to the controller's estimation because the system is sensorless.

TABLE I. RMS VALUES OF THE SPEED TRACKING ERRORS FOR BOTH CONTROL APPROACHES.

\begin{tabular}{|c|c|c|c|c|c|c|}
\hline \multirow{2}{*}{ Control } & \multicolumn{2}{|c|}{ Case-1 } & \multicolumn{2}{c|}{ Case-2 } & \multicolumn{2}{c|}{ Case-3 } \\
\cline { 2 - 7 } & $\begin{array}{c}\text { Speed } \\
\mathbf{m} / \mathbf{s}\end{array}$ & $\begin{array}{c}\text { Angle } \\
\mathbf{r a d} / \mathbf{s}\end{array}$ & $\begin{array}{c}\text { Speed } \\
\mathbf{m} / \mathbf{s}\end{array}$ & $\begin{array}{c}\text { Angle } \\
\mathbf{r a d} / \mathbf{s}\end{array}$ & $\begin{array}{c}\text { Speed } \\
\mathbf{m} / \mathbf{s}\end{array}$ & $\begin{array}{c}\text { Angle } \\
\mathbf{r a d} / \mathbf{s}\end{array}$ \\
\hline $\begin{array}{c}\text { PI- } \\
\text { MRAS }\end{array}$ & 0.0074 & 0.0413 & 0.0279 & 0.1032 & 0.0249 & 0.1012 \\
\hline $\begin{array}{c}\text { FO- } \\
\text { MRAS }\end{array}$ & 0.0051 & 0.020 & 0.0169 & 0.0272 & 0.0155 & 0.0241 \\
\hline
\end{tabular}

\section{CONCLUSIONS}

In this paper, an experimental study on the application of FO-MRAS controller to an electrically powered wheelchair platform under the different references has been presented for the first time in the literature. The model reference adaptive system (MRAS) technique has been presented to achieve the estimated value of system's velocity information. Complex reference are considered to control the velocity and direction angle of an EPW to verify the performance of the proposed FO-MRAS control method. Experimental results have verified that the vehicle's real velocity and direction angle are perfectly tracked and the estimation errors are small when compared the PI based MRAS method. On the other hand, the direction angle error of both controller has a similar behaviour in terms of using same reference between the wheel and the ground floor. The success of the proposed FO-MRAS method is due to the fractional order calculus feature. That is, fractional-order (FO) is an arbitrary order of the ordinary derivative and integral calculus that leads to more exibility and performance in the designing of the controller.

\section{REFERENCES}

[1] A. Yamashita, T. Arai, Jun Ota, H. Asama, "Motion planning of multiple mobile robots for Cooperative manipulation and transportation", in IEEE Trans. Robotics and Automation, vol. 19, no. 2, pp. 223-237, 2003. DOI: 10.1109/TRA.2003.809592.

[2] C. C. de Wit, O. J. Sordalen, "Exponential stabilization of mobile robots with nonholonomic constraints", in IEEE Trans. Automatic Control, vol. 37, no. 11, pp. 1791-1797, 1992. DOI: 10.1109/9.173153.

[3] U. Farooq, G. Abbas, S. O. Saleh, M. U. Asad, "Corridor navigation with fuzzy logic control for sonar based mobile robots", 7th IEEE Conf. Industrial Electronics and Applications (ICIEA 2012), Singapore, 2012, pp. 2087-2093. DOI: 10.1109/ICIEA.2012.6361074.

[4] Y. Kanayama, Y. Kimura, F. Miyazaki, T. Noguchi, "A stable tracking control method for an autonomous mobile robot", in Proc. IEEE Int. Conf. Robotics and Automation, Cincinnati, OH, 1990, pp. 384-389. DOI: 10.1109/ROBOT.1990.126006.

[5] W. Wu, H. Chen, Y. Wang, P. Woo, "Adaptive exponential stabilization of mobile robots with uncertainties", in Proc. 38th IEEE Conf. Decision and Control, Phoenix, AZ, 1999, pp. 3484-3489. DOI: 10.1109/CDC.1999.827870.

[6] J. Mu, X. G. Yan, S. K. Spurgeon, Z. Mao, "Generalized regular form based SMC for nonlinear systems with application to a WMR", in IEEE Trans. Industrial Electronics, vol. 64, no. 8, pp. 6714-6723, 2017. DOI: 10.1109/TIE.2017.2711500.

[7] A. Ailon, I. Zohar, "Control strategies for driving a group of nonholonomic kinematic mobile robots in formation along a timeparameterized path", in IEEE/ASME Trans. Mechatronics, vol. 17, no. 2, pp. 326-336, 2012. DOI: 10.1109/TMECH.2010.2103320.

[8] M. Aranda, G. Lopez-Nicolas, C. Sagues, Y. Mezouar, "Formation control of mobile robots using multiple aerial cameras", in IEEE Trans. Robotics, vol. 31, no. 4, pp. 1064-1071, 2015. DOI: 10.1109/TRO.2015.2452777.

[9] W. M. E. Mahgoub, I. M. H. Sanhoury, "Back stepping tracking controller for wheeled mobile robot", Int. Conf. Communication, Control, Computing and Electronics Engineering (ICCCCEE 2017), Khartoum, 2017, pp. 1-5. DOI: 10.1109/ICCCCEE.2017.7867663.

[10] J. X. Xu, Z. Q. Guo, T. H. Lee, "Design and implementation of integral sliding-mode control on an underactuated two-wheeled mobile robot", in IEEE Trans. Industrial Electronics, vol. 61, no. 7, pp. 3671-3681, 2014. DOI: 10.1109/TIE.2013.2282594.

[11] K. Sato, M. Yanagi, K. Tsuruta, "Adaptive Hळ trajectory control of nonholonomic mobile robot with compensation of input uncertainty", IEEE Int. Conf. Control Applications (CCA 2011), Denver, CO, 2011, pp. 699-705. DOI: 10.1109/CCA.2011.6044477.

[12] A. Skobeleva, V. Ugrinovskii, I. Petersen, "Extended Kalman filter for indoor and outdoor localization of a wheeled mobile robot", Australian Control Conf. (AuCC 2016), Newcastle, NSW, 2016, pp. 212-216. DOI: 10.1109/AUCC.2016.7868190.

[13] G. Peter, B. Kiss, G. Kovacs, "Kalman filter based cooperative landmark localization in indoor environment for mobile robots", IEEE Int. Conf. Systems, Man, and Cybernetics (SMC 2016), Budapest, 2016, pp. 1888-1893. DOI: 10.1109/SMC.2016.7844514.

[14] H. B. Azza, N. Zaidi, M. Jemli, M. Boussak, "Development and experimental evaluation of a sensorless speed control of SPIM using adaptive sliding mode-MRAS strategy", in IEEE Journal of Emerging and Selected Topics in Power Electronics, vol. 2, no. 2, pp. 319-328, 2014. DOI: 10.1109/JESTPE.2014.2299893.

[15] K. Shojaei, "Neural adaptive output feedback formation control of type (m, s) wheeled mobile robots", IET Control Theory \& Applications, vol. 11 , no. 4, pp. 504-515, 2017. DOI: 10.1049/ietcta.2016.0952

[16] M. Karimi-Ghartemani, M. Zamani, N. Sadati, M. Parniani, “An optimal fractional order controller for an AVR system using particle swarm optimization algorithm", Large Engineering Systems Conf. Power Engineering, Montreal, Que., 2007, pp. 244-249. DOI: 10.1109/LESCPE.2007.4437386.

[17] A. Al-Mayyahi, W. Wang, P. Birch, "Design of fractional-order controller for trajectory tracking control of a non-holonomic autonomous ground vehicle", Journal of Control, Automation and Electrical Systems, pp. 1-14, 2015. DOI: 10.1007/s40313-015-02142.

[18] H. Abu-Rub, A. Iqbal, J. Guzinski, High performance control of AC drives with MATLAB/Simulink models. John Wiley \& Sons, Hoboken, NJ, 2012. DOI: 10.1002/9781119969242.

[19] M. Stulrajter, V. Hrabovcova, M. Franko, "Permanent magnets synchronous motor control theory", J. Elect. Eng, vol. 58, no. 2, pp. 79-84, 2007.

[20] H. B. Azza, N. Zaidi, M. Jemli, M. Boussak, "Development and experimental evaluation of a sensorless speed control of SPIM using adaptive sliding mode-MRAS strategy", IEEE Journal of Emerging and Selected Topics in Power Electronics, vol. 2, no. 2, pp. 319-328, 2014. DOI: 10.1109/JESTPE.2014.2299893.

[21] V. Minambres-Marcos, M. A. Guerrero-Martinez, E. RomeroCadaval, J. Gutierrez, "Issues and improvements of hardware/software co-design sensorless implementation in a permanent magnet synchronous motor using Veristand", IEEE Int. Symposium on Sensorless Control for Electrical Drives and Predictive Control of Electrical Drives and Power Electronics (SLED/PRECEDE 2013), Munich, 2013, pp. 1-7. DOI: 10.1109/SLED-PRECEDE.2013.6684523. 